\title{
SARS-CoV-2 und "Chilblains": Dokumente einer leichten Infektion?
}

Bei allen Patienten mit frostbeulenartigen Läsionen während der COVID-19-Pandemie fiel die PCR zum Zeitpunkt der dermatologischen Diagnose negativ aus. Bei $30 \%$ waren allerdings Antikörper gegen SARS-CoV-2 nachweisbar. Histologische und biochemische Untersuchungen weisen auf eine Typ-I-Interferonopathie hin.

Während der COVID-19-Pandemie wird immer wieder über Patienten mit frostbeulenartigen Läsionen (Chilblain-like Lesions) berichtet. Um der Pathophysiologie und einem möglichen Zusammenhang näherzukommen, haben Dr. Thomas Hubiche von der Université Côte d'Azur, Nizza, und Kollegen Personen mit entsprechenden Manifestationen in einer prospektiven Fallserie in ihrer Klinik untersucht [1].

In die Studie eingeschlossen wurden 40 konsekutive Patienten mit frostbeulenartigen Läsionen. Die meist jungen Patienten im Durchschnittsalter von 22 Jahren wurden allgemeinärztlich und dermatologisch untersucht.

Meist befanden sich die Hautveränderungen an den Zehen, selten an den Fersen oder Fingern. Der Verlauf bei den einzelnen Patienten war sehr ähnlich: Zunächst traten Juckreiz und Schmerzen auf, dann bildeten sich pink-rote Papula oder Plaques, die sich zu violetten Läsionen, häufig mit Blasen und Nekrosen entwickelten. Letztere zeigten sich bei elf der Patienten, bei 19 bestand eine Akrozyanose oder die Zehnen waren kalt. Alle Hauterscheinungen heilten zwar vollständig ab, zum Teil klagten die Patienten aber noch wochenlang über kalte und blaugefärbte Zehen.

\section{SARS-CoV-2-Antikörper bei jedem dritten Patienten}

Bis zu sechs Wochen vor dem Auftreten der „Pseudofrostbeulen “ hatten sich bei elf der Patienten klinische Hinweise auf eine mögliche COVID-19-Erkrankung gezeigt. Durchschnittlich waren 18,5 Tage bis zum Auftreten der „Pseudo- frostbeulen“ vergangen. Es fanden sich zwei familiäre Cluster, wo die Hauterscheinungen zur gleichen Zeit bestanden. In der PCR bestätigte sich bei keiner der Personen mit Chilblains eine aktuelle SARS-CoV-2-Infektion. Allerdings fiel bei zwölf Studienteilnehmern die SARS-CoV-2-Serologie positiv aus (1 Patient IgM-positiv, 8 Patienten IgA-positiv und 5 Patienten lgG-positiv).

\) Meist befanden sich die Hautveränderungen an den Zehen, selten an den Fersen oder Fingern

Am häufigsten traten pathologische Laborwerte bei den D-Dimeren auf. Dieser Wert war bei 24 Patienten

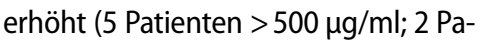
tienten $>2000 \mu \mathrm{g} / \mathrm{ml}$ ). Antinukleäre Antikörper wurden bei neun und Antiphospholipid-Antikörper bei vier Patienten nachgewiesen. Der Test auf Kryoglobuline und die ParvovirusB19-Serologie fielen bei allen Studienteilnehmern negativ aus.

Die histologischen Präparate ließen Zeichen einer lymphozytären Entzündung sowie Gefäßschäden in Form von verdickten Venolenwänden und Perizytenhyperplasie erkennen. Nach einer In-vitro-Stimulation konnte gegenüber PCR-positiven Patienten mit moderater bis schwerer akuter SARS-CoV-2-Infektion ein signifikanter Anstieg der IFN-alpha-Produktion beobachtet werden. Dabei zeigte sich kein Unterschied zwischen denen, die SARS-CoV-2-Antikörper entwickelt hatten, und denen, deren Serologie negativ blieb.
Diesen Befunden zufolge schreiben Hubiche und Kollegen die frostbeulenartigen Hautveränderungen, die während der COVID-19-Pandemie immer wieder beschrieben werden, einer virusinduzierten Typ-I-Interferonopathie zu. Auf dieser Basis sei ein kausaler Zusammenhang zwischen den Hauterscheinungen und einer SARS-CoV-2-Infektion zwar nicht bewiesen, es habe aber auch keine andere Ursache für die „Pseudofrostbeulen“ festgestellt werden können, so die Studienautoren. Eine zentrale Rolle der Typ-I-Interferone für die Immunantwort auf SARS-CoV-2- wird derzeit intensiv diskutiert. [2,3]

\section{Literatur}

1. Hubiche T, Cardot-Leccia N, Le Duff F, et al (2020) Clinical, Laboratory, and InterferonAlpha Response Characteristics of Patients With Chilblain-like Lesions During the COVID-19 Pandemic. JAMA Dermatol. https://doi.org/10.1001/jamadermatol.2020. 4324

2. Bastard et al (2020) Autoantibodies against type I IFNs in patients with life-threatening COVID-19. Science 370

3. Zhang et al (2020) Inborn errors of type I IFN immunity in patients with life-threatening COVID-19. Science 370

Hinweis des Verlags. Der Verlag bleibt in Hinblick auf geografische Zuordnungen und Gebietsbezeichnungen in veröffentlichten Karten und Institutsadressen neutral.

hautnah $2021 \cdot 20: 12-13$

https://doi.org/10.1007/s12326021-00427-9

(c) Springer-Verlag GmbH Austria, ein Teil von Springer Nature 2021 
Hier steht eine Anzeige.

Springer 